\begin{tabular}{|c|c|c|c|c|c|c|}
\hline \multirow{4}{*}{ Impact Factor: } & ISRA (India) & $=3.117$ & SIS (USA) & $=0.912$ & ICV (Poland) & $=6.630$ \\
\hline & ISI (Dubai, UAE & $=0.829$ & РИНЦ (Russia & $=0.156$ & PIF (India) & $=1.940$ \\
\hline & GIF (Australia) & $=0.564$ & ESJI (KZ) & $=8.716$ & IBI (India) & $=4.260$ \\
\hline & JIF & $=1.500$ & SJIF (Morocco & $=5.667$ & OAJI (USA) & $=0.350$ \\
\hline
\end{tabular}

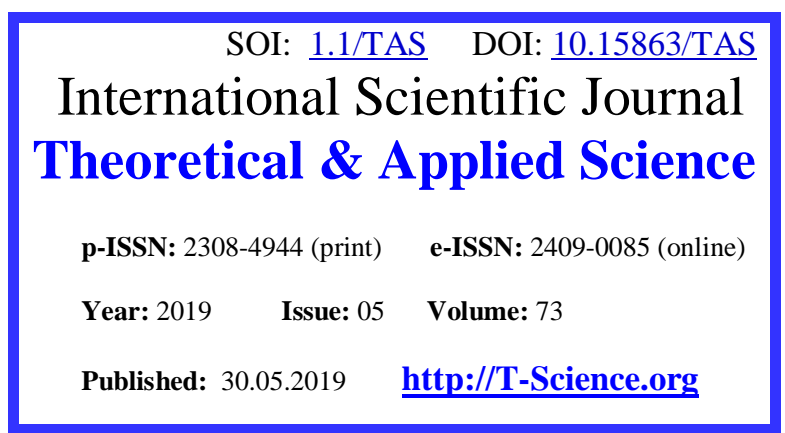

SECTION 4. Computer science, computer engineering and automation.
QR - Issue

QR - Article
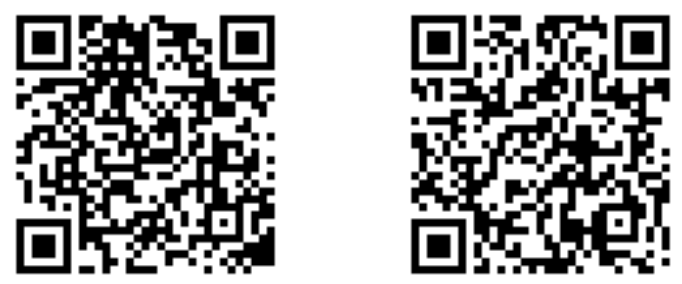

Adlet Sakenovich Alipbekov

Master of Technical Sciences,

Software Engineer of the Educational Process in the

Distance Learning Department (DLD),

Taraz State University named after M.Kh. Dulaty,

Kazakhstan

alipbekov@outlook.com

Zhanar Tursinbaevna Aldashova

Bachelor of Technical Sciences,

Software Engineer of the Registrar Office,

Taraz State University named after M.Kh. Dulaty,

janny.90-16@mail.ru

Anastassiya Andreevna Makovetskaya Bachelor of Technical Sciences,

Software Engineer of the Educational Process in the

Distance Learning Department (DLD)

Taraz State University named after M.Kh. Dulaty, aytsan.m@bk.ru

Saltanat Shadiyarbekkyza Dulatbaeva Master of Technical Sciences, Teacher of Computer science,

Taraz State University named after M.Kh. Dulaty, dulatbaevasaltanat@mail.ru

Murat Sauranbayevich Tulenbayev Doctor of Technical Sciences, professor of Computer science,

Taraz State University named after M.Kh. Dulaty, mtulenbaev@mail.ru

\title{
FEATURES OF THE USE OF MOBILE ANDROID APPLICATIONS IN THE EDUCATIONAL ENVIRONMENT
}

Abstract: Modern mobile devices (gadgets) use various operating systems, such as Android and IOS. Among them, the Android OS occupies a leading position, as it is the most accessible and convenient. This article discusses the architecture and mobile applications of the Android OS, providing training in a modern educational system, provides a comparative analysis of efficiency.

Key words: online learning, distance learning, online courses, online mass courses, mobile applications, mobile application development, mobile applications market analysis

Language: Russian

Citation: Alipbekov, A. S., Aldashova, Z. T., Makovetskaya, A. A., Dulatbaeva, S. S., \& Tulenbayev, M. S. (2019). Features of the use of mobile android applications in the educational environment. ISJ Theoretical \& Applied Science, 05 (73), 479-483.

Soi: http://s-o-i.org/1.1/TAS-05-73-73 Doi: crossef https://dx.doi.org/10.15863/TAS.2019.05.73.73 


\begin{tabular}{|c|c|c|c|c|c|c|}
\hline \multirow{4}{*}{ Impact Factor: } & ISRA (India) & $=3.117$ & SIS (USA) & $=0.912$ & ICV (Poland) & $=6.630$ \\
\hline & ISI (Dubai, UAE & $=0.829$ & РИНЦ (Russia & $=0.156$ & PIF (India) & $=1.940$ \\
\hline & GIF (Australia) & $=0.564$ & ESJI (KZ) & $=8.716$ & IBI (India) & $=4.260$ \\
\hline & JIF & $=1.500$ & SJIF (Morocce & $=5.667$ & OAJI (USA) & $=0.350$ \\
\hline
\end{tabular}

\section{ОСОБЕННОСТИ ИСПОЛЬЗОВАНИЯ МОБИЛЬНЫХ ANDRОID ПРИЛОЖЕНИЙ В ОБРАЗОВАТЕЛЬНОЙ СРЕДЕ}

Аннотация: B современных мобильных устройствах (гаджетах) используются различные операционные системы, такие как Androidu IOS. Среди них OC Androidзанимает лидирующие позиции, так как является наиболее доступной и удобной. В данной статье рассматриваются архитектура и мобильные приложения OC Android, обеспечивающие обучение в современной образовательной системе, дается сравнительный анализ эффективности.

Ключевые слова: онлайн обучение, дистанцичонное обучение, онлайн курсы, онлайн массовые курсы, мобильные приложения, разработка мобильных приложений, анализ рынка мобильных приложений.

\section{Introduction}

Парадигма «обучение через всю жизнь с технологией доступной в любом месте и в любое время» является актуальной платформой современного образовательного процесса. Реализация этой платформы в образовательной системе стало возможным в связи бурным развитием цифровых технологий и устройств, таких как интернет, компьютеры, мобильные устройства. Сегодня мобильные устройства и мобильные технологии совершают революционные изменения в методах и технологиях обучения, заставляя пересматривать концептуальные основы системы образования во всем мире .

Операционная система Android была разработана Энди Рубином, Ричем Майнером, Ником Сирсом и Крисом Уайтом в 2003 году. В последние годы Android привлекает большое внимание в развивающемся сегменте образовательных технологий, на данной ОС уже разработано более 100000 образовательных приложений.Androidприложением может быть любая образовательная информация разработанная для мобильных устройств под управлением ОC Android, которая обеспечивает удобство и доступ для учащихся из любого места. По проведенным анализам, у учащихся благодаря использованию образовательных технологии наблюдался рост интереса к обучению, креативность. Мобильные приложения позволяют вмещать в себя сразу несколько областей обучения, и дает доступ к ним из любого места. Повсеместной учебной средой является любая обстановка, в которой учащиеся могут полностью погрузиться в учебный процесс. Поскольку мобильные устройства поддерживают обучение в любое время и в любом месте, мобильное обучение с использованием Android может способствовать развитию системы образования. Платформа приложения обучения Android позволяет учащемуся получить доступ к учебной информации и взаимодействовать с другими учениками, будто они находятся рядом, в то время, когда он дома либо в другом отдаленном месте. Использование портативных вычислительных устройств (таких как ноутбуки, планшетные ПК и смартфоны на базе Android) с беспроводными сетями обеспечивает мобильность и обучение, позволяя преподаванию и обучению выходить за пределы традиционной формы обучения в учебных классах. Из-за развития современных мобильных устройств увеличивается количество и мобильных приложений, разработанных связанных с образованием. Последние мобильные аппаратные и программные платформы благодаря 4G позволяют запускать более быстрые и объемные приложения [1].

\section{Materials and Methods}

Обучение с помощью платформы Androidэто форма цифрового обучения, которая может применяться в целях обучения и преподавания, некоторые эксперты в области образования рассматривают его как подмножество электронного обучения, но с глубоким содержанием, которое дает доступ прямиком на мобильные Android устройства. Преподавание и обучение с использованием платформы Androidможно легко реализовать без оснащения тяжелыми оборудованиями. Есть несколько факторов, которые создают мобильную компьютерную базу. Во-первых, операционная система Android бесплатно предоставляется для мобильных устройств, что делает разработку и установку приложений очень простой. Во-вторых, существует большая база учебных материалов и контента, которая продолжает расширяться и к ней имеют доступ как студенты, так и преподаватели. Например, студенты могут загружать и практиковаться в коротких тестах на своих мобильных устройствах где присутствует мгновенная обратная связь, которая, предоставляет информацию для лучшего понимания. Данный тип обучения стремительно набирает актуальность, что в свою очередь привлекает молодое поколение. Кроме того, студенты напрямую могут загружать заметки с веб-сайта Google Doc, используя платформу Android.B настоящее время технология позволяет учащимся совместно использовать и редактировать документы онлайн. Таким образом, концепция коллективного интеллекта превратилась из абстрактной концепции в 


\begin{tabular}{|c|c|c|c|c|c|c|}
\hline \multirow{4}{*}{ Impact Factor: } & ISRA (India) & $=3.117$ & SIS (USA) & $=0.912$ & ICV (Poland) & $=6.630$ \\
\hline & ISI (Dubai, UAI & $=0.829$ & РИНЦ (Russia & $=0.156$ & PIF (India) & $=1.940$ \\
\hline & GIF (Australia) & $=0.564$ & ESJI (KZ) & $=8.716$ & IBI (India) & $=4.260$ \\
\hline & JIF & $=1.500$ & SJIF (Morocco & $=5.667$ & OAJI (USA) & $=0.350$ \\
\hline
\end{tabular}

осязаемую реализацию в образовательной сфере [2].

Android - операционная система для мобильных устройств и ноутбуков, а также платформа для приложений, поддерживаемая Google, a архитектура Android обучения является дополнением к платформе мобильных приложений. Предоставляя открытую платформу разработки, Android предоставил разработчикам возможность создавать чрезвычайно насыщенные и инновационные приложения с очень богатым набором пользовательских интерфейсов, которые поддерживают широкий диапазон форматов аудио и видеофайлов. Разработчики и производители мобильных телефонов по всему миру приняли эту платформу из-за ее открытого исходного кода и разнообразных возможностей работы, а также разработки приложений с использованием языков программирования, таких как Java и Kotlin.

Архитектура обучения Android, показанная на рисунке 1, реализует положения новой парадигмы образования, которая предполагает «обучение в любое время и в любом месте». Обучение можно проводить как в традиционных

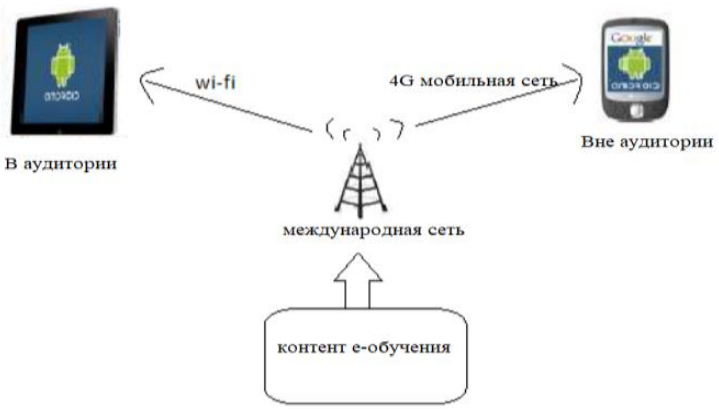

Рисунок 1. Блок-схема архитектуры с использованием Android

классных комнатах, так и вне классной комнаты, например, в автобусе или дома с использованием проводной и беспроводной сети с $4 \mathrm{G}$ и локальной сетью для постоянного подключения к университетскому серверу с архитектурой webслужб. Интерактивное обучающее приложение, в котором оба клиентских модуля работают на мобильных устройствах, подходящих для самых разных сред обучения, позволяет студентам получать знания из того, что они видят, слышат, читают и воспринимают [3].

Образовательные приложения дают возможность учащимся использовать свое мобильное устройство для получения образовательных преимуществ в доступное для них время независимо от их местоположения. Мобильные телефоны Android являются не просто дополнительным оборудованием для развитых стран, но и могут играть важную роль в их системах образования. Мобильные Android устройства обеспечивают доступ к огромному количеству образовательных и учебных ресурсов. В развивающихся странах мобильные устройства Android могут легко компенсировать ограниченный доступ к Интернету и доступ к данным, что, в свою очередь, способствует развитию их инфраструктуры и образования.

Android Learning- это приложение электронного обучения, которое предоставляет учебные контенты и материалы для обучения с помощью устройств беспроводной связи. Электронное обучение дает возможность собирать исследовательские данные по предмету. Использование Android Learning для образовательных и социальных сетей и коммуникаций более распространено, чем для разработки пользовательских приложений - 38,2\% внедряют, разрабатывают или готовят учебные материалы для социальных сетей и только 24,7\% для пользовательских приложений и разработок. $71,2 \%$ респондентов используют свои мобильные устройства в коммерческих целях. Из тех, кто проводил внедрение Android Learning, $50 \%$ получали положительные результаты (табл. $1)$.

\section{Conclusion}

Несмотря на некоторые ограничения возможностей мобильных устройств и мобильных приложений, популярность Android растет, около $29 \%$ студентов учатся с помощью программного обеспечения для Android. Bce больше образовательных учреждений и компании переходят на разработку мобильных приложений для Android,так как они просты в установке, более надежны и удобны для обучающихся, влияние мобильных приложений приобретает глобальный характер [4]. 


\begin{tabular}{|c|c|c|c|c|c|c|}
\hline \multirow{4}{*}{ Impact Factor: } & ISRA (India) & $=3.117$ & SIS (USA) & $=0.912$ & ICV (Poland) & $=6.630$ \\
\hline & ISI (Dubai, UAE & $=0.829$ & РИНЦ (Russia & $=0.156$ & PIF (India) & $=1.940$ \\
\hline & GIF (Australia) & $=0.564$ & ESJI (KZ) & $=8.716$ & IBI (India) & $=4.260$ \\
\hline & JIF & $=1.500$ & SJIF (Morocce & $=5.667$ & OAJI (USA) & $=0.350$ \\
\hline
\end{tabular}

Таблица 1. Сравнение видов обучения

\begin{tabular}{|l|l|l|l|}
\hline & $\begin{array}{l}\text { Традиционное } \\
\text { образование }\end{array}$ & $\begin{array}{l}\text { Электронное } \\
\text { образование }\end{array}$ & Android образование \\
\hline Доступ & Ограниченный & $24 / 7$ & $24 / 7$ \\
\hline Качество & меняющийся & последовательный & $\begin{array}{l}\text { Последовательно- } \\
\text { прогрессивный }\end{array}$ \\
\hline Запоминаемость & меняющийся & $\begin{array}{l}\text { Высокая } \\
\text { запоминаемость, } \\
\text { персонализированное } \\
\text { обучение }\end{array}$ \\
\hline
\end{tabular}

Кроме того мобильные приложения эффективно и дешево расширяют сферу охвата образования в каждой части мира [5]. Это, в свою очередь, повышает престиж университетов, тем самым улучшая образовательные результаты и доходы [6]. Таким образом, использование Android приложений в образовательных целях увеличивается, это иллюстрирует диаграмма представленная на рисунке 2.

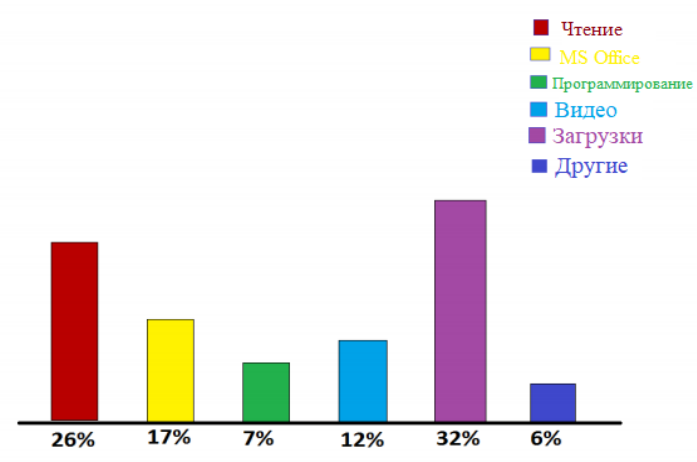

Рисунок 2. График использования Android приложений

Кроме того, использование мобильных приложений обеспечивает персонифицированное образование. Это позволяет учащимся контролировать предмет, время и способы обучения. Таким образом, увеличивается количество источников, расширяющих сотрудничество и социальные сети среди студентов [7]. В сущности, Android приложения способствуют высокому уровню взаимодействия между студентами без посредничества и модерации инструктора, что позволяет учащимся самостоятельно развивать свои собственные идеи, навыки, знания, обучающую сеть, усиливать самовыражение [8], [9], [10], глубокое обучение и абстрактная концептуализация [11], [12]. Однако применение Android приложений разностороннее и охватывает многие сферы жизнедеятельности человека, что показано в соответствии с рисунком 3.
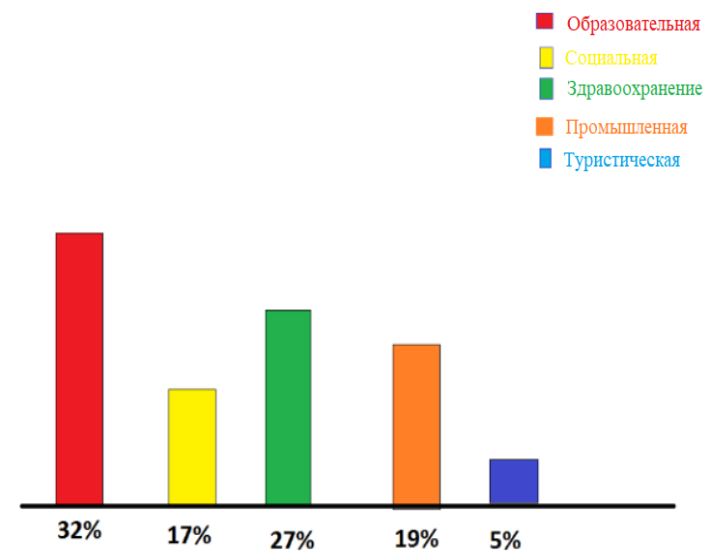

Рисунок 3. График сфер применения Android приложений 


\begin{tabular}{llllll} 
& ISRA (India) $=\mathbf{3 . 1 1 7}$ & SIS (USA) & $=\mathbf{0 . 9 1 2}$ & ICV (Poland) & $=\mathbf{6 . 6 3 0}$ \\
Impact Factor: & ISI (Dubai, UAE) $=\mathbf{0 . 8 2 9}$ & PUHL (Russia) $=\mathbf{0 . 1 5 6}$ & PIF (India) & $=\mathbf{1 . 9 4 0}$ \\
& GIF (Australia) $=\mathbf{0 . 5 6 4}$ & ESJI (KZ) & $=\mathbf{8 . 7 1 6}$ & IBI (India) & $=\mathbf{4 . 2 6 0}$ \\
& JIF & $\mathbf{1 . 5 0 0}$ & SJIF (Morocco) $=\mathbf{5 . 6 6 7}$ & OAJI (USA) & $\mathbf{0 . 3 5 0}$ \\
\hline
\end{tabular}

\section{References:}

1. (2015). Dokument ob issledovanii operacionnoj sistemy Android $\mathrm{s}$ tochki zreniya udovletvorennosti pol'zovatelej. Mezhdunarodnyj zhurnal peredovyh tekhnologij v oblasti inzhenerii i nauki Tom № 03, vypusk № 01, yanvar' 2015.

2. (2013). Uchebno-tekhnicheskaya razrabotka dlya kamery na baze oruzhiya, mezhdunarodnyj zhurnal nauchno-tekhnicheskih issledovanij, tom 2, vypusk 5, maj 2013 goda

3. (n.d.). Android, «Versii dlya platform». Retrieved 2019, from http://developer.android.com/resources/dashbor $\mathrm{d} /$ platformversin

4. (n.d.). Proektirovanie i razrabotka mobil'nogo prilozheniya dlya sovmestnogo obucheniya $\mathrm{s}$ ispol'zovaniem Android, Informacionnyj zhurnalTekhnologiya i primenenie $\mathrm{V}$ obrazovanii.

5. Sultan, N. (2014). Cloud and MOOKS: the servitization of it and, $\|$ vol. 1, no. 2, pp.1-15.

6. Hollands, F., \& Tirthali, D. (2014). MOOKs: Expectations and Reality,\| Full Rep., no. May, pp.1- 208.

7. Chacón-Beltrán, R. (2014). Massive Online Open Courses and Language Learning: the Case for a Beginners' English Course,\| Procedia Soc. Behav. Sci., vol. 141, pp. 242-246.

8. Mackness, R., Mak, J., \& Williams, S. (2012). The ideals and reality of participating in a MOOK. In L. Dirckinck-Holmfeld et al. (Eds.),॥ in Proceedings of the 7th International Conference on Networked Learning, pp. 266275.

9. McAuley, D., Stewart, A., Siemens, B., \& Cormier, G. (2010). Massive open online courses digital ways of knowing and learning.

10. Levy, S., \& Schrire, D. (2012). The case of a massive open online course at a college of education. Retrieved July 14, 2016, from: http://conference.nmc.org/files/smkbMOOK.pd $\mathrm{f}$

11. Vonderwell, K., Liang, S., \& Alderman, X. (2007). Asynchronous discussions and assessment in online learning. J. Res. Technol. Educ., vol. 39, no. (3), pp.309-328.

12. Aragon, N., Johnson, S., \& Shaik, S. (2002). The influence of learning style preferences on student success in online versus face-to-face environments. Am. J. Distance Educ., vol. 16, no. 4, pp. 227-244. 